\title{
II ritmo dialitico bisettimanale può essere utilizzato in pazienti selezionati all'avvio del trattamento emodialitico cronico
}

Giornale di Tecniche Nefrologiche e Dialitiche 2018, Vol. 30(2) 100-104

(C) The Author(s) 2018

Article reuse guidelines:

sagepub.com/journals-permissions DOI: $10.1177 / 0394936218766533$

journals.sagepub.com/home/gtn

(S)AGE

\author{
Marina Cornacchiari, Francesco Cosa, Valentina Martina, \\ Luca Di Toma, Barbara Gidaro, Bianca Visciano, Antonella Stasi, \\ Maria Meneghini, Annalisa Neri and Carlo Guastoni
}

Twice-weekly emodialysis can be scheduled for patients selected at the start of the dialysis treatment. In recent years, several studies have evaluated the differences in survival and residual renal function between patients who underwent two times weekly haemodialysis (HDB) and three times weekly haemodialysis (HDT). In most cases, these studies have taken into account only the patient survival but not the time into treatment or "technique survival". In our centre we have retrospectively evaluated the incident patients that were treated with HDB for at least three months between 0 I January 2013 and 0 I November 20I5, focusing on the HDB technique survival. During the follow-up, 35 patients started treatment with HDB, while 13 with HDT. On average, patients treated with HDT were younger than HDB patients $(60.9 \pm 15.9$ years vs. $77.8 \pm 9.6$ years, $p=0.01)$, and body weight was higher in HDT patients $(7 \mathrm{I} \pm 8 \mathrm{~kg}$ vs. $63 \pm \mathrm{l} 3 \mathrm{~kg}, \mathrm{p}=0.0 \mathrm{l})$. HDT patients were more frequently affected by two or more comorbidities (diabetes, peripheral vasculopathy, cardiac disease, chronic obstructive lung disease) than patients treated by HDB. The creatinine clearance $(\mathrm{CrCl})$ evaluated at the beginning of treatment was similar in the two groups $(7.48 \pm 5 \mathrm{~mL} /$ min in HDB vs $5.4 \pm 5 \mathrm{~mL} / \mathrm{min}$ in HDT, $\mathrm{p}=\mathrm{NS}$ ). Urine output at the beginning of the study was higher in the HDB than the HDT group $(1,785 \pm 480 \mathrm{~mL} / 24 \mathrm{~h}$ vs. $530 \pm 500 \mathrm{~mL} / 24 \mathrm{~h}, \mathrm{p}=0.00 \mathrm{l})$. Results. The mean survival of the HDB was

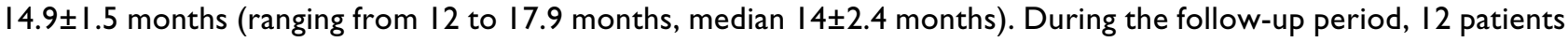
switched from HDB to HDT due to the presence of signs of fluid overload in ten of them. In these patients the $\mathrm{CrCl}$ and the urine output were significantly reduced by the end of the treatment $(\mathrm{CrCl}$ was $3.4 \pm 1.9 \mathrm{~mL} / \mathrm{min} \mathrm{vs} 6.8 \pm 2 \mathrm{~mL} /$ $\min , p=0.01$; urine output was $995 \pm 567 \mathrm{~mL} / 24 \mathrm{~h}$ vs $1400 \pm 400 \mathrm{~mL} / 24 \mathrm{~h}, \mathrm{p}=0.02$ ). No hospital admissions for uremic symptoms (pericarditis, pulmonary oedema, severe hyperkalaemia) were recorded throughout the follow-up period in HDB patients. Conclusions. Our study shows that HDB can be proposed in selected patients for a considerable period. Decrease of urine output and presence of symptoms related to fluid overload are the two main factors leading to the switch to HDT treatment.

Parole chiave

HDB (haemodialysis biweekly), HDT (haemodialysis triweekly), RRF (residual renal function)

Gli autori non hanno ricevuto sponsorizzazioni economiche per la preparazione dell'articolo.

\section{Introduzione}

Nella pratica clinica di molti centri per la dialisi peritoneale viene utilizzata una strategia di dose incrementale ${ }^{1}$. Ciò significa che la dialisi viene iniziata con un numero ridotto di scambi giornalieri, che successivamente viene incrementato al ridursi della funzione renale residua (residual renal function, RRF).
Per l'emodialisi cronica, le linee guida NKF-KDOQI² considerano proponibile il poter iniziare il trattamento con un ritmo bisettimanale quando si è in presenza di un filtrato renale residua $\geqslant 3 \mathrm{~mL} / \mathrm{min}$.

Azienda Socio Sanitaria Territoriale Ovest Milanese - Ospedale Di Legnano E Magenta

\section{Corrispondenza:}

Carlo Guastoni, Azienda Socio Sanitaria Territoriale Ovest Milanese

- Ospedale Di Legnano E Magenta.

Email: carlo.guastoni@asst-ovestmi.it 
Tabella I. Caratteristiche dei due gruppi di pazienti: HDB, emodialisi bisettimanale; HDT, emodialisi trisettimanale; early referral, pazienti con follow-up predialitico $\geqslant 6$ mesi. Nel testo sono riportati la presenza e il numero di comorbilità.

\begin{tabular}{llllllll}
\hline & Età & $\begin{array}{l}\text { Early } \\
\text { refferral }\end{array}$ & $\begin{array}{l}\text { Late } \\
\text { referral }\end{array}$ & Peso & $\begin{array}{l}\text { I-2 } \\
\text { comorbilità }\end{array}$ & $\begin{array}{l}2-3 \\
\text { comorbilità }\end{array}$ & $\begin{array}{l}\geqslant 4 \\
\text { comorbilità }\end{array}$ \\
\hline HDT & $77.8 \pm 9.6$ & 17 & 18 & $63 \pm 16 \mathrm{Kg}$ & 13 & 20 & 1 \\
& & $(48.5 \%)$ & $(51.5 \%)$ & & $(38 \%)$ & $(59 \%)$ & $(3 \%)$ \\
HDB & $60.9 \pm 15.9$ & 67 & 6 & $71 \pm 8 \mathrm{Kg}$ & 1 & $(2)$ & 0 \\
& & $(53.8 \%)$ & $(46.2 \%)$ & & $(8 \%)$ & $(92 \%)$ & $(0 \%)$ \\
\hline
\end{tabular}

Negli ultimi anni diversi studi hanno valutato l'efficacia della dialisi bisettimanale (HDB), paragonandola alla emodialisi trisettimanale (HDT), in termini di sopravvivenza dei pazienti e andamento della funzione renale residua ${ }^{3,4}$. Tuttavia, in questi studi spesso manca la valutazione di un aspetto fondamentale, vale a dire la durata del trattamento HDB (permanenza del paziente in HDB).

Nel nostro centro la strategia di dialisi incrementale viene frequentemente utilizzata nei pazienti incidenti in dialisi peritoneale. Questa esperienza ci ha indotto ad utilizzare un'iniziale prescrizione bisettimanale nei pazienti incidenti in emodialisi cronica privi di sintomi uremici e/o di segni di overload di fluidi.

In questi pazienti, il passaggio successivo ad un ritmo trisettimanale è dettato dalla presenza di sintomi clinici o biochimici (overload di fluidi, riduzione della diuresi e della funzione renale residua, insufficiente depurazione).

In questo studio abbiamo voluto valutare retrospettivamente la nostra casistica di pazienti incidenti, dal 01 gennaio 2013 al 01 novembre 2015, trattati con HDB per almeno tre mesi, focalizzandoci in particolare sulla durata della tecnica HDB.

\section{Casistica e metodi}

Nel periodo di osservazione, 35 pazienti hanno iniziato e proseguito per almeno tre mesi il trattamento con HDB, mentre 13 hanno iniziato direttamente con HDT. Il follow-up medio è stato di 13.2 \pm 7.6 mesi (3-34.3 mesi).

In tabella 1 sono riassunte le caratteristiche dei due gruppi di pazienti.

Entrambi i gruppi sono stati trattati con bicarbonato dialisi: membrana polisulfone o poliamide high flux, superficie di $1.6 \mathrm{~m}^{2}$ (30 pazienti in HDB e 8 pazienti in HDT) oppure $2 \mathrm{~m}^{2}$ (5 pazienti in HDB e 5 pazienti in HDT), QB (flusso ematico) $300 \mathrm{~mL} / \mathrm{min}$, QD (flusso dialisato) $500 \mathrm{~mL} / \mathrm{min}$.

La composizione del bagno dialisi era la stessa nelle due metodiche $\left(\mathrm{Na}^{+} 140 \mathrm{mMol} / \mathrm{L}, \mathrm{K}^{+} 2-3 \mathrm{mMol} / \mathrm{L}, \mathrm{HCO}_{3}{ }^{-}\right.$ $34 \mathrm{mMol} / \mathrm{L}$, Acetato $3 \mathrm{mMol} / \mathrm{L}, \mathrm{Mg}^{2+} 0.5 \mathrm{mMol} / \mathrm{L}^{-C^{2+}}$ $1.5 \mathrm{mMol} / \mathrm{L}$, glucosio $1 \mathrm{~g} / \mathrm{L}$ ).

I pazienti incidenti in HDT avevano un'età media inferiore rispetto a quella dei pazienti in HDB (60.9 15.9 anni vs $77.8 \pm 9.6$ anni, $\mathrm{p}=0.01$ ). Il peso corporeo era, in media, maggiore nei pazienti trattati con HDT $(71 \pm 8 \mathrm{~kg}$ vs
$63 \pm 13 \mathrm{~kg}, \mathrm{p}=0.01$ ); i pazienti early referral (ovvero con follow-up predialitico $\geqslant 6$ mesi) erano il $48.5 \%$ in HDB e il $53.8 \%$ in HDT ( $\mathrm{p}=\mathrm{NS}$ ).

I pazienti in HDT erano più frequentemente affetti da due o più comorbidità (diabete, vasculopatia periferica, cardiopatia, broncopneumopatia cronica ostruttiva) rispetto ai pazienti trattati con HDB.

La percentuale dei portatori di FAV (fistola alterovenosa) o di CVC (catetere venoso centrale) non era statisticamente differente nei due gruppi di pazienti.

La clearance della creatinina (creatinine clearance, $\mathrm{CrCl}$ ), misurata nelle urine e nel plasma 24 ore precedentemente al primo ciclo di emodialisi, era simile nei due gruppi $(7.48 \pm 5 \mathrm{~mL} / \mathrm{min}$ in $\mathrm{HDB}$ vs $5.4 \pm 5 \mathrm{~mL} / \mathrm{min}$ in HDT, $\mathrm{p}=\mathrm{NS}$ ) (Figura 1).

Dopo l'inizio del trattamento dialitico (HDT e HDB) tutti i pazienti hanno avuto una dieta libera. In particolare, ai pazienti che hanno iniziato con HDB non è stato richiesto di ridurre l'apporto proteico $\mathrm{e} / \mathrm{o}$ fosforico, specialmente considerando che già l'età avanzata in sé avrebbe potuto comportare una riduzione del loro apporto alimentare.

Durante il follow-up sono stati eseguiti i controlli mensili del dei valori di urea, creatinina, calcio, fosforo, albumina, emoglobina $(\mathrm{Hb})$, paratormone $(\mathrm{PTH}) \mathrm{Kt} / \mathrm{V}$.

La diuresi media in 24 ore al momento dell'inserimento in dialisi cronica era superiore nei pazienti trattati con HDB $(1,785 \pm 480 \mathrm{~mL})$ rispetto a quelli trattati con HDT $(530 \pm 500 \mathrm{~mL}, \mathrm{p}=0.001)$ (figura 1).

\section{Risultati}

La permanenza media dei pazienti in HDB è stata di $14.9 \pm 1.5$ mesi (range 12-17.9 mesi) con una mediana di 14 \pm 2.4 mesi (9.2-18.8).

In figura 2 la curva di Kaplan-Meier mostra la sopravvivenza dei pazienti nel gruppo HBD.

Durante il periodo di osservazione, 10 pazienti sono passati dalla HDB alla HDT, a causa della presenza di segni di overload di fluidi (5 pazienti) e per la riduzione della diuresi al di sotto di $500 \mathrm{~mL} / 24$ ore (5 pazienti).

In tabella 2 sono mostrati i dati di laboratorio all'inizio ed alla fine dell'osservazione nei due gruppi di pazienti. Tutti i parametri valutati (creatinina, urea, albumina, $\mathrm{Hb}$, $\mathrm{PTH}$, calcio, fosforo e $\mathrm{Kt} / \mathrm{V}$ ) non mostrano differenze statisticamente significative tra i due gruppi, ad eccezione 


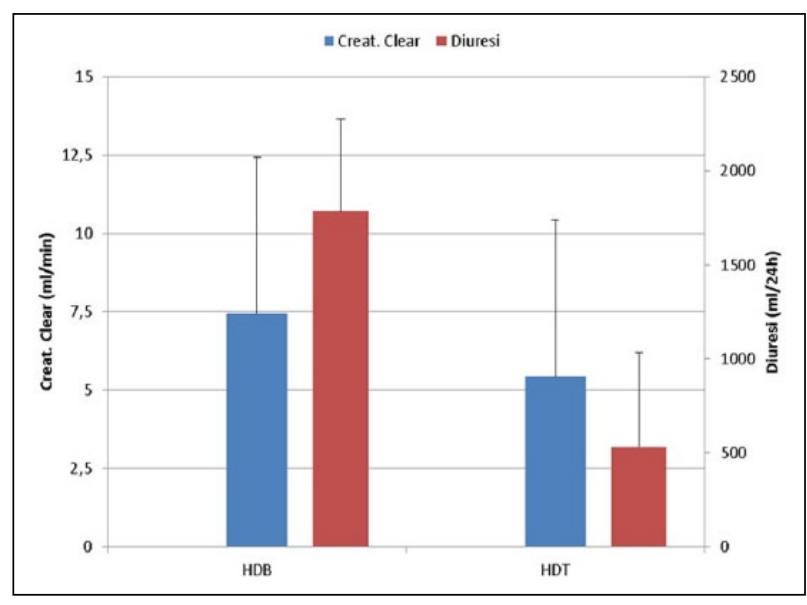

Figura I. La $\mathrm{CrCl}$ e diuresi all'inizio del trattamento emodialitico nei due gruppi di pazienti. La diuresi nel gruppo HDB era di $1,785 \pm 480 \mathrm{~mL} / 24$ ore, e nel gruppo HDT $530 \pm 500$ $\mathrm{mL} / 24$ ore $(p=0.00 \mathrm{I})$. La $\mathrm{CrCl}$ nel gruppo HDB era di $7.8 \pm 5$ $\mathrm{mL} / \mathrm{min}$ e nel gruppo HDT $5.4 \pm 5 \mathrm{~mL} / \mathrm{min}(\mathrm{p}=\mathrm{NS})$.

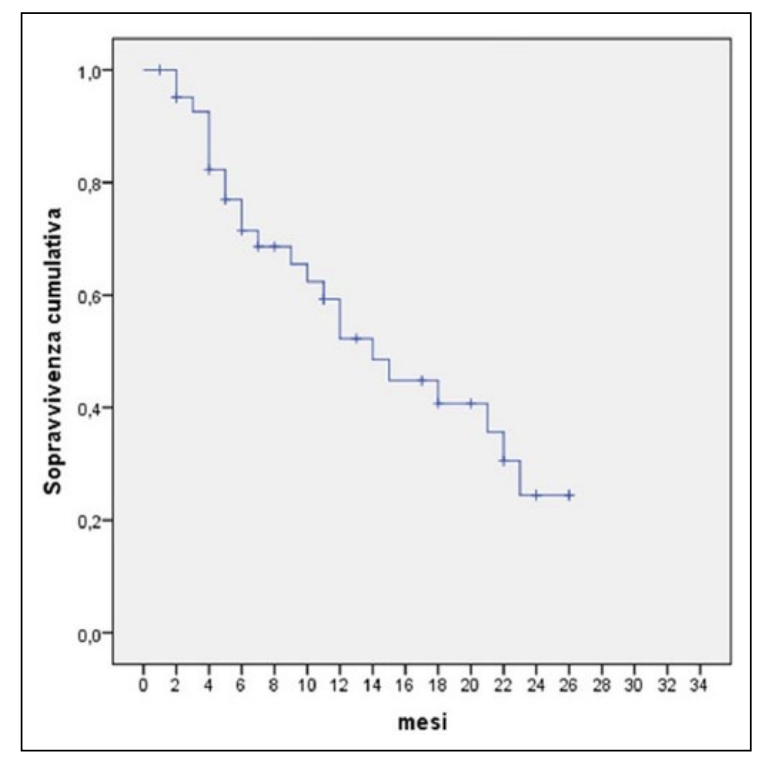

Figura 2. Curva di Kaplan-Meier che indica la sopravvivenza cumulativa dei pazienti che hanno iniziato il trattamento dialitico cronico col metodo HDB.

del livello di albumina che è significativamente maggiore nei pazienti del gruppo HDT all'inizio del trattamento $(\mathrm{p}=0.03)$.

La presenza di indici biochimici di insufficiente depurazione (elevati valori di urea e/o creatinina, cattivo controllo del fosforo, iperparatiroidismo) ha motivato il passaggio di 2 pazienti al gruppo HDT.

In 23 mesi, 9 pazienti nel gruppo HDB e 3 nel gruppo HDT sono deceduti ( $\mathrm{p}=\mathrm{NS})$.

$\mathrm{La} \mathrm{CrCl}$ e la diuresi residua media nei 12 pazienti che sono passati alla HDT si sono ridotte significativamente rispetto all'inizio del trattamento $(\mathrm{CrCl}$ da $3.4 \pm 1.9 \mathrm{~mL} / \mathrm{min}$ a $6.8 \pm 2 \mathrm{~mL} / \mathrm{min}, \mathrm{p}=0.01$ ); diuresi residua media da $995 \pm 567$ $\mathrm{mL} /$ die a $1,400 \pm 400 \mathrm{~mL} /$ die, $\mathrm{p}=0.02$ ).

Rispetto ai 12 pazienti che sono passati alla HDT, i 23 pazienti che hanno continuato la HDB presentavano, al termine del periodo di osservazione, una $\mathrm{CrCl}$ simile $(4.22 \pm 2.8 \mathrm{~mL} / \mathrm{min}$ vs $3.4 \pm 1.9 \mathrm{~mL} / \mathrm{min}$; $\mathrm{p}=\mathrm{NS})$ ed una diuresi media significativamente maggiore $(1,357 \pm 568$ $\mathrm{mL} /$ die vs $995 \pm 567 \mathrm{~mL} /$ die; $\mathrm{p}=0.01$ ) (figura 3 ).

Durante il follow-up nessuno dei pazienti in HDB è stato ricoverato per sintomatologia uremica (pericardite, edema polmonare, iperkaliemia grave).

\section{Discussione}

Nei paesi occidentali la frequenza delle sedute dialitiche nei pazienti che iniziano il trattamento sostitutivo cronico è quasi sempre standardizzata a tre sedute settimanali, indipendentemente dalle condizioni cliniche e dalla funzione renale residua del paziente ${ }^{5}$. La dialisi bisettimanale in Europa è infatti utilizzata in una bassa percentuale di pazienti, come dimostrato dallo studio DOPPS ${ }^{6}$. L'utilizzo della HDB è invece molto più diffuso nei paesi in via di sviluppo, in particolare in Cina dove la HDB viene usata nel $26 \%$ dei casi, prevalentemente a causa dello stile di vita, peso corporeo e abitudini alimentari dei pazienti in dialisi cronica, oltre che in conseguenza alla necessità del contenimento dei costi del trattamento dialitico ${ }^{4}$.

In realtà, nella pratica quotidiana, è frequente in dialisi peritoneale la prescrizione di una dose dialitica incrementale, vale a dire l'inizio con una dose dialitica ridotta e il successivo aumento della dose in conseguenza al deterioramento della funzione renale residua ${ }^{1}$. Un approccio analogo è stato proposto anche per i pazienti incidenti in trattamento emodialitico cronico utilizzando un ritmo dialitico bisettimanale, quando è presente una sufficiente funzione renale residua.

La dialisi bisettimanale è infatti considerata proponibile dalle linee guida del Disease Outcomes Quality Initiative (DOQI) del 2006 per i pazienti con funzione renale residua $^{2}$, misurata con la media della $\mathrm{CrCl}$ e dell'urea endogena, $R R F, \geqslant 3 \mathrm{~mL} / \mathrm{min} / 1.73 \mathrm{~m}^{2}$. Le più recenti NKF$\mathrm{DOQI}^{7}$ raccomandano, come indice di adeguatezza dialitica, un Kt/V settimanale dell'urea (RRF+ clearance dialitica "standard" o "equivalente") di $\geqslant 2.1^{7,8}$.

Diversi studi hanno valutato l'uso della dialisi bisettimanale/infrequente, e quasi tutti hanno posto attenzione al dato della sopravvivenza e all'andamento della funzione renale residua ${ }^{9}$.

Recentemente Kalantar-Zadeh et al. ${ }^{10}$ hanno proposto il concetto di "transitioning hemodialysis" e di "incremental dialysis" definendo i possibili criteri di selezione per utilizzare una strategia di dose incrementale in emodialisi (tabella 3).

Recentemente, Obi et al. ${ }^{11}$ hanno riportato i risultati di uno studio retrospettivo su una coorte di pazienti incidenti in emodialisi. Nel loro studio, 2 gruppi di pazienti, selezionati per paragonabili caratteristiche cliniche, 
Tabella 2. Valori di creatinina, urea, albumina, emoglobina $(\mathrm{Hb})$, paratormone (PTH), calcio totale, fosfato, e Kt/V all'inizio ed alla fine dell'osservazione nei gruppi HDB e HDT.

\begin{tabular}{|c|c|c|c|c|c|c|}
\hline & Inizio (HDB) & Fine (HDB) & $p$ & Inizio (HDT) & Fine (HDT) & $p$ \\
\hline Creatinina (mg/dL) & $6.9 \pm 2$ & $7.7 \pm 2$ & ns & $7.3 \pm 3$ & $8.4 \pm 2$ & ns \\
\hline Urea (mg/dL) & $168 \pm 58$ & $160 \pm 53$ & ns & $|5| \pm 42$ & $148 \pm 45$ & ns \\
\hline Albumina (g/dL) & $3.3 \pm 0.5$ & $3.2 \pm 0.5$ & 0.03 & $3.7 \pm 0.4$ & $3.1 \pm 0.5$ & ns \\
\hline $\mathrm{Hb}(\mathrm{g} / \mathrm{dL})$ & $\mid I .1 \pm 1.3$ & $11.2 \pm 1.1$ & ns & $10 . \pm 1.4$ & $10.9 \pm 1.3$ & ns \\
\hline PTH (pg/mL) & $378 \pm 247$ & $256 \pm 202$ & ns & $264 \pm 242$ & $340 \pm 263$ & ns \\
\hline Calcio (mg/dL) & $8.4 \pm 0.9$ & $8.3 \pm 0.7$ & ns & $8.8 \pm 0.9$ & $8.9 \pm 0.8$ & ns \\
\hline Fosforo (mg/dL) & $5.1 \pm 1.9$ & $4.7 \pm 1.7$ & ns & $4.2 \pm 1.9$ & $4.7 \pm 1.7$ & ns \\
\hline $\mathrm{Kt} / \mathrm{V}$ & & $1.07 \pm 0.19$ & ns & & $1.1 I \pm 0.2$ & ns \\
\hline
\end{tabular}

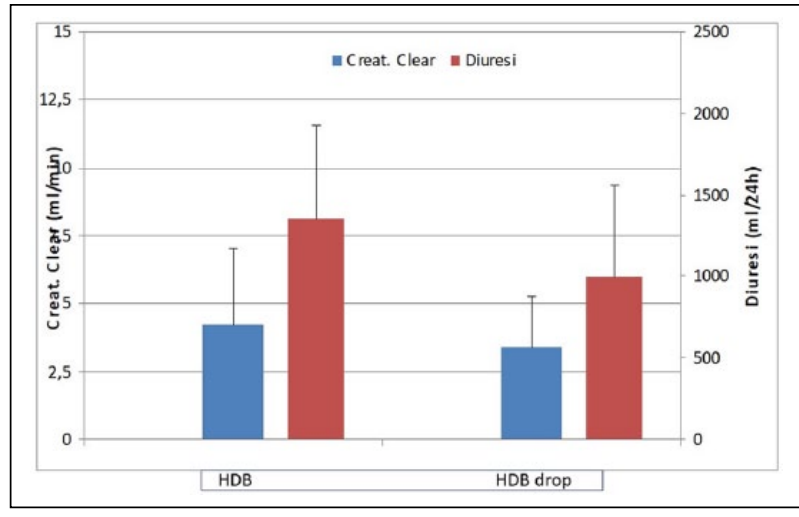

Figura 3. La figura mostra la $\mathrm{CrCl}$ (misurata sulle urine delle 24 ore e sulla creatininemia predialitica) e la diuresi/24 ore alla fine del periodo di osservazione nei 23 pazienti che hanno mantenuto la HDB e nei 12 che sono passati alla HDT (HDB drop). $\mathrm{CrCl} p=n s$, diuresi $p=0.001$.

Tabella 3. Criteri di selezione dei pazienti possibili candidati alla dialisi bisettimanale. Modificata da Kalantar-Zadeh K. e colleghilo.

I. Buona funzione renale residua con diuresi $>0.5 \mathrm{~L} / \mathrm{d}$

2. Ridotto sovraccarico idrosalino tra 2 trattamenti emodialitici con un incremento

ponderale $<2.5 \mathrm{~kg}$ (o meno del $5 \%$ del peso secco ideale)

3. Massa corporea appropriate alla funzione renale residua

4. Iperkaliemia (potassio $>5.5 \mathrm{mEq} / \mathrm{L}$ ) rara

5. Iperfosfatemia (fosfato $>5.5 \mathrm{mg} / \mathrm{dL}$ ) rara

6. Buono stato nutrizionale

7. Soddisfacente qualità della vita in relazione allo stato di salute

biochimiche ed età, sono stati confrontati (351 pazienti sottoposti a HDB e 8,068 pazienti sottoposti a HDT).

Il gruppo trattato con HDB ha evidenziato nel follow-up una minore riduzione della RRF andare a capo. Riguardo l'effetto della dialisi sulla RRF, è stato evidenziato come la dialisi peritoneale sia associata ad un suo maggiore mantenimento nel tempo. Questo effetto, evidenziato anche con la HDB, può essere associato alla minore frequenza degli episodi di ischemia renale che comportano una progressiva perdita della funzione renale residua e che sono correlati alle rapide rimozioni di volume che si verificano durante le sedute ${ }^{12,13,14}$.

Va aggiunto che un ritmo dialitico più "intensivo" può anche ridurre lo stimolo all'iper-funzionamento dei nefroni residui secondo la teoria di Bricker ${ }^{15}$.

Nello studio di Obi et al. ${ }^{11}$ il rischio di morte nel gruppo HDB era statisticamente simile a quello del gruppo HDT solo nel gruppo di pazienti con $R R F \geqslant 3 \mathrm{~mL} / \mathrm{min} / 1.73 \mathrm{~m}^{2}(\mathrm{p}=0.3)$. I pazienti nel gruppo HDB con una diuresi giornaliera $\leqslant 600$ $\mathrm{mL}$ e un incremento ponderale interdialitico $\geqslant 6 \%$ mostravano una sopravvivenza peggiore rispetto ai pazienti nel gruppo HDT. Gli autori hanno concluso che l'emodialisi due volte alla settimana può essere sicura nei pazienti in emodialisi incidenti con buona RRF e con incrementi ponderali contenuti.

Negli USA l'effetto del ritmo dialitico sulla sopravvivenza è stato valutato da Mathew et al. ${ }^{16}$, il quale ha confrontato 434 pazienti trattati in HDB con 50,162 pazienti in HDT. La sopravvivenza è risultata migliore in HDT solo nei pazienti caratterizzati da un elevato indice di comorbilità (indice di Charlson >5).

Un aspetto importante riguardo l'applicabilità dell'emodialisi incrementale nella pratica clinica è quello della permanenza in trattamento HDB. I nostri risultati hanno dimostrato che la HDB ha avuto una durata media di oltre un anno, senza che nel follow-up si siano evidenziati ricoveri dovuti a sintomi correlati al cattivo controllo dello stato uremico (edema polmonare, iperkaliemia).

Questa permanenza media in HDB è senza dubbio un dato ragguardevole, in particolare se consideriamo che la RRF all'inizio del trattamento non era diversa da quella dei pazienti che sono stati inseriti direttamente in HDT.

Va considerato inoltre che la RRF è stata valutata sulla base della $\mathrm{CrCl}$ endogena che, come è noto, sovrastima il reale valore della funzione renale nel paziente uremico rispetto al valore ottenuto con la media della $\mathrm{CrCl}$ sommata a quella dell'urea ${ }^{17}$. Questa considerazione avvalora il fatto che la durata della HDB non è stata condizionata da un inizio precoce del trattamento.

Essendo lo studio retrospettivo non è stato possibile valutare la clearance residua dell'urea, al fine di misurare in maniera più corretta il filtrato renale residuo, tuttavia va sottolineato che la $\mathrm{CrCl}$ all'inizio del trattamento non era significativamente differente nei due gruppi. 
Indubbiamente l'aspetto più importante rimane quello della scelta dei pazienti che possono iniziare il trattamento cronico con una HDB. Nella nostra casistica i pazienti incidenti nel gruppo HDB sono stati selezionati in base ad una minore comorbilità e ad un minore peso corporeo associati ad una diuresi giornaliera significativamente maggiore rispetto a quelli trattati con HDT.

La maggiore età anagrafica ha rappresentato un altro criterio di scelta a favore della HDB. Il fattore età va indubbiamente considerato perché i pazienti più anziani sono quelli che possono trarre i maggiori vantaggi da un trattamento bisettimanale. Infatti, iniziare con un ritmo dialitico bisettimanale comporta un minor numero di spostamenti da casa all'ospedale e contribuisce a rendere psicologicamente meno pesante l'impatto della dialisi cronica. Entrambi questi aspetti incidono positivamente sulla qualità di vita, soprattutto dei pazienti più anziani.

Nella nostra casistica il passaggio ad un ritmo trisettimanale è stato motivato dalla riduzione significativa della diuresi associata alla presenza di segni di overload di fluido nell' $83 \%$ dei casi (10/12 casi).

A questo proposito un limite del nostro studio consiste nel non aver valutato se la riduzione della diuresi fosse correlata al maggior incremento ponderale interdialitico ed alla conseguente maggiore rimozione di volume durante le sedute.

Viceversa, la necessità di ottenere un trattamento dialitico più efficiente ha motivato il passaggio alla HDT in $2 / 12$ pazienti $(17 \%)$; questo dato potrebbe essere correlato con la selezione dei pazienti con un peso corporeo ridotto. Va sottolineato come la natura retrospettiva dello studio non ci abbia permesso di valutare l'eventuale correlazione tra l'utilizzo dei due ritmi dialitici differenti ed il peso corporeo e/o gli indici nutrizionali dei pazienti.

Infine, va ricordato che una strategia dialitica incrementale in emodialisi permette di poter sfruttare con maggiore efficienza i posti tecnici e di trattare un numero maggiore di pazienti rispetto alla strategia "standard" basata su un ritmo trisettimanale costante per tutti i pazienti.

Questo ultimo aspetto può rappresentare un importante vantaggio di fronte al costante incremento della domanda di dialisi cronica a cui si assiste in molte nostre regioni.

\section{Dichiarazione di assenza di conflitto di interessi}

Gli Autori dichiarano di non avere conflitti di interessi.

\section{Finanziamenti}

Gli Autori dichiarano di non aver ricevuto finanziamenti specifici da qualsiasi ente nei settori pubblico, privato o senza fini di lucro.

\section{Bibliografia}

1. Neri L, Viglino G, Marinangeli G., Rocca AR, Laudon A, Ragusa A, Cabiddu G, Peritoneal Dialysis Study Group of Italian Society of Nephrology. Incremental Start to PD as Experienced in Italy: Results of Censuses Carried out from 2005 to 2014. Journal of Nephrology 2017; 4: 593-99.
2. KDOQI, and National Kidney Foundation. KDOQI Clinical Practice Guidelines and Clinical Practice Recommendations for Anemia in Chronic Kidney Disease. American Journal of Kidney Diseases 2006; 5 Suppl 3: S11-145.

3. Wong J, Vilar E, Davenport and Farrington K. Incremental Haemodialysis. Nephrology, Dialysis, Transplantation 2015; 10: 1639-48.

4. Bieber B, Qian J, Anand S, Yan Y, et al. Two-Times Weekly Hemodialysis in China: Frequency, Associated Patient and Treatment Characteristics and Quality of Life in the China Dialysis Outcomes and Practice Patterns Study. Nephrology, Dialysis, Transplantation 2014; 9: 1770-77.

5. Rhee CM, Unruh M, Chen J, Kovesdy CP, Zager P and Kalantar-Zadeh K. Infrequent Dialysis: A New Paradigm for Hemodialysis Initiation. Seminars in Dialysis 2013; 6: 720-27.

6. Pisoni RL, Gillespie BW, Dickinson DM, Chen K, Kutne MH and Wolfe RA. The Dialysis Outcomes and Practice Patterns Study (DOPPS): Design, Data Elements, and Methodology. American Journal of Kidney Diseases 2004; 5 Suppl 2: 7-15.

7. National Kidney Foundation. KDOQI Clinical Practice Guideline for Hemodialysis Adequacy: 2015 Update. American Journal of Kidney Diseases 2015; 5: 884-930.

8. Daugirdas JT, Depner TA, Greene T, Levin NW, Chertow GM and Rocco MV. Frequent Hemodialysis Network Trial Group. 'Standard Kt/Vurea: A Method of Calculation That Includes Effects of Fluid Removal and Residual Kidney Clearance. Kidney International 2010; 7: 637-44.

9. Minmin Z, Wang M, Li H, Yu P, Yuan L, Hao C, Chen J and Kalantar-Zadeh K. Association of Initial Twice-Weekly Hemodialysis Treatment with Preservation of Residual Kidney Function in ESRD Patients. American Journal of Nephrology 2014; 2: 140-50.

10. Kalantar-Zadeh K, Unruh M, Zager PG, et al. Twice-Weekly and Incremental Hemodialysis Treatment for Initiation of Kidney Replacement Therapy. American Journal of Kidney Diseases. 2014; 2: 181-86.

11. Obi Y, Eriguchi R, Ou SM, Rhee CM and Kalantar-Zadeh K. What Is Known and Unknown About Twice-Weekly Hemodialysis. Blood Purification 2015; 4: 298-305.

12. Diao Z, Zhang D, Dai W., Ding J, Zhang A and Liu W. Preservation of residual renal function with limited water removal in hemodialysis patients. Ren Fail 2011; 33: 875-877.

13. Lin YF, Huang JW, Wu MS, et al. Comparison of residual renal function in patients undergoing twice-weekly versus three-times-weekly haemodialysis. Nephrology (Carlton) 2009; 14: 59-64.

14. Daugirdas JT, Greene T, Rocco MV, et al. Effect of frequent hemodialysis on residual kidney function. Kidney International 2013; 83 (5): 949-58.

15. Bricker NS, Morrin PA and Kime SW Jr. The pathologic physiology of chronic bright's disease. An exposition of the "intact nephron hypothesis". American Journal of Medicine 1960; 28: 77-98.

16. Mathew A, Yoshitsugu O, Rhee CM, et al. Treatment Frequency and Mortality among Incident Hemodialysis Patients in the United States Comparing Incremental with Standard and More Frequent Dialysis. Kidney International 2016; 5: 1071-79.

17. Casino FG. The grey line of dialysis initiation: as early as possible that is, by the incremental modality. Giornale Italiano Di Nefrologia 2010; 6: 574-83. 